\title{
Osimertinib in poor performance status patients with T790M-positive advanced non-small-cell lung cancer after progression of first- and second-generation EGFR-TKI treatments (NEJ032B)
}

\author{
Yukari Tsubata $^{1}(1) \cdot$ Kana Watanabe $^{2} \cdot$ Ryota Saito $^{3} \cdot$ Atsushi Nakamura $^{4} \cdot$ Hiroshige Yoshioka $^{5} \cdot$ Mami Morita $^{2}$. \\ Ryoichi Honda $^{6} \cdot$ Nobuhiro Kanaji $^{7} \cdot$ Satoshi Ohizumi ${ }^{8} \cdot$ Daisuke Jingu $^{9} \cdot$ Taku Nakagawa $^{10} \cdot$ Kensuke Nakazawa $^{11}$. \\ Atsuto Mouri $^{12}$. Susumu Takeuchi ${ }^{13} \cdot$ Naoki Furuya $^{14} \cdot$ Yuki Akazawa $^{15} \cdot$ Kiyotaka Miura $^{16} \cdot$ Eiki Ichihara $^{17}$. \\ Makoto Maemondo ${ }^{18} \cdot$ Satoshi Morita ${ }^{19} \cdot$ Kunihiko Kobayashi $^{12} \cdot$ Takeshi Isobe $^{1}$
}

Received: 16 June 2021 / Accepted: 28 September 2021 / Published online: 13 October 2021

(c) The Author(s) 2021

\begin{abstract}
Background Osimertinib is effective in patients with T790M mutation-positive advanced non-small-cell lung cancer (NSCLC) resistant to epidermal growth factor receptor (EGFR) tyrosine kinase inhibitors (TKIs). However, its effectiveness and safety in patients with poor performance status (PS) are unknown.

Methods Enrolled patients showed disease progression after treatment with gefitinib, erlotinib, or afatinib; T790M mutation; stage IIIB, IV, or recurrent disease; and PS of 2-4. Osimertinib was orally administered at a dose of $80 \mathrm{mg} / \mathrm{day}$. The primary endpoint of this phase II study (registration, jRCTs061180018) was response rate and the secondary endpoints were progression-free survival (PFS), overall survival (OS), disease control rate, and safety.

Results Thirty-three patients were enrolled, of which $69.7 \%$ and $24.2 \%$ had PS of 2 and 3, respectively. One patient was excluded due to protocol violation; in the remaining 32 patients, the response rate was $53.1 \%$; disease control rate was $75.0 \%$; PFS was 5.1 months; and OS was 10.0 months. The most frequent adverse event of grade 3 or higher severity was lymphopenia (12.1\%). Interstitial lung disease (ILD) was observed at all grades and at grades 3-5 in 15.2\% (5/33) and 6.1\% (2/33) of patients, respectively. Treatment-related death due to ILD occurred in one patient. Patients negative for activating EGFR mutations after osimertinib administration had longer median PFS than those positive for these mutations.

Conclusion Osimertinib was sufficiently effective in EGFR-TKI-resistant, poor PS patients with T790M mutation-positive advanced NSCLC. Plasma EGFR mutation clearance after TKI treatment could predict the response to EGFR-TKIs.
\end{abstract}

Keywords EGFR T790M · Non-small-cell lung cancer · Osimertinib $\cdot$ Phase II $\cdot$ Poor performance status

\section{Introduction}

Epidermal growth factor receptor $(E G F R)$ gene mutations are the most common driver oncogene mutations associated with non-small cell lung cancer (NSCLC), accounting for $55 \%$ of driver oncogene mutations in lung adenocarcinoma cases in East Asia [1]. The recommended treatments for stage IV EGFR-positive lung cancer are EGFRtyrosine kinase inhibitor (TKI) monotherapy, EGFR-TKI plus cytotoxic combination chemotherapy, and EGFR-TKI

Yukari Tsubata

ytsubata@med.shimane-u.ac.jp

Extended author information available on the last page of the article plus anti-angiogenic combination therapy [2]. A promising response rate (RR) and prolongation of progression-free survival (PFS) have been reported for each of these treatments [3-7]. Nonetheless, disease progression is observed after 9-21 months in almost all patients who respond to treatment [3-7]. The EGFR T790M mutation is considered a cause of acquired resistance to EGFR-TKI therapy and is found in approximately $60 \%$ of patients with lung adenocarcinoma treated with EGFR-TKIs [8, 9].

Osimertinib is a third-generation EGFR-TKI. A clinical trial comparing osimertinib with pemetrexed plus either carboplatin or cisplatin in patients with EGFR T790M mutation-positive NSCLC and with disease progression after first-line therapy reported significantly longer PFS (10.1 months vs. 4.4 months) and significantly better 
response rates ( $71 \%$ vs. 31\%) with osimertinib [10]. Favorable outcomes have also been achieved with osimertinib, as the first-line therapy in patients with EGFR-positive stage IV NSCLC [11]. Notably, osimertinib has been shown to be effective in patients with central nervous system (CNS) metastasis based on a subgroup analysis of such patients [12]. Therefore, osimertinib is a key drug for EGFR-positive patients with CNS metastases. Osimertinib is used as a standard therapy in patients with performance status (PS) scores of 0-1 [2]. In addition, specific TKI therapy for the driver oncogene mutation is recommended for patients with mutation-positive lung cancer but a PS score of 2 due to the demonstrated efficacy in patients with good PS and the likelihood of a good response [2]. For patients with PS scores of 3-4, best supportive care is indicated, and aggressive anticancer treatment is not recommended. A first-generation EGFR-TKI, gefitinib, was efficacious in patients with EGFR-positive lung cancer and poor PS [13], but the clinical utility and safety of osimertinib, a third-generation EGFRTKI, remain unclear.

Therefore, we conducted an open-label, multicenter, single-arm phase II study to evaluate the effectiveness and safety of osimertinib in patients with EGFR T790M mutation-positive advanced NSCLC with Eastern Cooperative Oncology Group (ECOG) PS scores of 2-4.

\section{Patients and methods}

\section{Patients}

The main eligibility criteria were as follows:

- Non-radiocurable stage IIIB, IIIC, IVA, or IVB, or postoperative recurrent NSCLC confirmed either histologically or cytologically

- Positive for an EGFR-sensitizing mutation (G719X, exon 19 deletion, exon 21 L858R point mutation, or exon 21 L861Q point mutation)

- Imaging-confirmed disease progression after treatment with a first- or second-generation EGFR-TKI (gefitinib, erlotinib, or afatinib)

- EGFR T790M mutation confirmed in a specimen collected after disease progression following the most recent treatment regimen (all methods used to determine the EGFR T790M mutation status were accepted)

- Aged 20 years or older at the time of informed consent

- ECOG PS score of 2-4, with the performance decline determined to be due to lung cancer by the attending physician

Patients who received multiple EGFR-TKIs or used vascular endothelial growth factor inhibitors or cytotoxic chemotherapy in combination with an EGFR-TKI were also eligible.

The main exclusion criteria were as follows:

- History of interstitial lung disease (ILD), drug-induced ILD, radiation pneumonitis requiring steroid therapy, or evidence of an active ILD

- Previous immune checkpoint inhibitor treatment

- Clinically unstable brain metastasis

- Abnormal electrocardiogram, prolonged QTc, or a factor increasing the risk of induced arrhythmia

\section{Study design and treatment}

NEJ032B was a multicenter, single-arm, phase 2 study assessing the efficacy and safety of osimertinib in patients with EGFR T790M-positive NSCLC. The study was conducted in 17 institutions across Japan from February 2017 to May 2019.

Patients with confirmed EGFR T790M-positive lung cancer received $80 \mathrm{mg}$ of oral osimertinib once daily. Patients received the treatment until progressive disease (PD), unacceptable toxicity, or consent withdrawal. Until the fourth week after the start of treatment, the condition of patients and results of laboratory tests were examined weekly. CT imaging was performed at least every 8 weeks, and confirmation was performed 4 weeks later in patients in whom complete response (CR) or partial response (PR) was confirmed. The treatment efficacy was assessed using the Response Evaluation Criteria in Solid Tumors (RECIST), version 1.1. Adverse events were evaluated using the Common Terminology Criteria for Adverse Events (CTCAE), version 4.0. The efficacy and safety assessments were conducted by the Central Effectiveness and Safety Assessment Committee.

\section{Plasma sample collection and EGFR mutation analysis}

The NEJ032B biomarker study was conducted in patients who consented to the biomarker study. The plasma ctDNA analysis to detect the activating EGFR mutations and T790M mutation was performed using an improved PNALNA Polymerase Chain Reaction (PCR) clamp method (LSI Medience Corporation, Tokyo, Japan). Whole blood samples $(21 \mathrm{~mL})$ were collected in ethylenediaminetetraacetic acid (EDTA) tubes before TKI treatment (P0), 8 weeks after the initiation of study treatment (P1), and after disease progression (P2). Samples were mixed thoroughly, and the plasma isolated by centrifuging blood at $2000 \times g$ for $10 \mathrm{~min}$ was stored at $-20{ }^{\circ} \mathrm{C}$. DNA was extracted from plasma samples using the QIAamp Circulating Nucleic Acid kit. PCR primers were designed to 
amplify G719X, exon 19 deletion, T790M, L858R, and L861Q. LNA probes were prepared complementary to each mutant allele, and PNA clamps were complementary to the respective wild-type alleles.

\section{Statistical analysis}

Statistical analyses were performed using Statistical Analysis System version 9.4 (SAS Institute Inc., North Carolina, USA). The primary endpoint was RR, and secondary endpoints were disease control rate, time to treatment failure, PFS, overall survival (OS), PS improvement, safety, and tolerability.

Osimertinib therapy has a reported RR of $63.6 \%$ in EGFR T790M-positive patients [14]. For the present study, an RR of $25 \%$ was considered a clinically meaningful threshold for patients with inoperable or recurrent NSCLC. Assuming a decrease of approximately $10 \%$ based on previous study results in patients with good PS, the anticipated RR was set at $50 \%$. Given an $\alpha$-error of 0.025 (one-sided) and $\beta$-error of 0.2 , the required number of patients was determined to be 29 . Based on this and allowing for dropouts, the target number of patients was set at 32. The most informative secondary endpoint to clinical status was PS improvement, which was defined as the proportion of per-protocol patients whose PS during osimertinib treatment was improved from baseline. PFS was defined as the interval between the months relapsed form the day of enrollment and the date of the first observation of disease progression or death from any cause. Patients who were alive without disease progression at the data cutoff point (May 21, 2020) were censored at the last point, as the patients were assessed to be progression-free. PFS and OS were estimated using the Kaplan-Meier method.

\section{Ethics}

This study was conducted in accordance with the principles of the Declaration of Helsinki and the Good Clinical Practice Guidelines. All patients provided written informed consent. The study protocol conformed with the Clinical Trials Act of 2017, was approved by the certified clinical research review board of Shimane University, and is published on the Japan Registry of Clinical Trials (jRCTs061180018).

\section{Results}

\section{Patient characteristics}

Thirty-three patients were enrolled in the study between February 2017 and May 2019. The primary endpoint RR was calculated in a per-protocol set of 32 patients because one patient violated the protocol; the patient received a prohibited concomitant therapy (radiotherapy). All other endpoints, including safety, were analyzed in the full analysis set. The median age of the enrolled patients was 72 (47-89) years, and most patients were women (27 patients, $81.8 \%$ ). The most common PS score was 2 (23 patients, 69.7\%), and the most common previous treatment was EGFR-TKI monotherapy (18 patients, 54.5\%) (Table 1).

\section{Efficacy}

In the per-protocol set of 32 patients, the primary endpoint RR was 53.1\% (95\% confidence interval [CI]: 34.7-70.9),
Table 1 Patient demographic and clinical characteristics at baseline

\begin{tabular}{ll}
\hline Total $(N)$ & 33 \\
\hline Median age (range) & $72(47-89)$ \\
Sex (male/female) $(\%)$ & $6 / 27(18.2 / 81.8)$ \\
ECOG PS 2/3/4 (\%) & $23 / 8 / 2(69.7 / 24.2 / 6.1)$ \\
Clinical stage before starting protocol treatment: IVA/IVB/postoperative & $7 / 23 / 3(21.2 / 69.7 / 9.1)$ \\
recurrence (\%) & \\
Prior treatment & $18(54.5)$ \\
EGFR-TKI alone & $7(21.2)$ \\
EGFR-TKI/cytotoxic anticancer agent/another molecularly targeted drug & $6(18.2)$ \\
EGFR-TKI/cytotoxic anticancer agent & $2(6.1)$ \\
EGFR-TKI/another molecularly targeted drug & $16 / 6 / 3 / 8(48.5 / 18.2 / 9.1 / 24.2)$ \\
Line of treatment: second/third/fourth/fifth or later $(\%)$ & $33(100)$ \\
Histopathological classification: adenocarcinoma (\%) & $16(48.5)$ \\
Brain metastasis: present $(\%)$ &
\end{tabular}

ECOG PS Eastern Cooperative Oncology Group performance status, EGFR-TKI epidermal growth factor receptor tyrosine kinase inhibitor 
Table 2 Response rate and disease control rate

\begin{tabular}{ll}
\hline Total $(N)$ & 32 \\
\hline Complete response (\%) & $2(6.3)$ \\
Partial response (\%) & $15(46.9)$ \\
Stable disease (\%) & $7(21.9)$ \\
Progressive disease (\%) & $5(15.6)$ \\
Non-evaluable $(\%)$ & $3(9.4)$ \\
Response rate $(\%, 95 \% \mathrm{CI})$ & $17(53.1,34.7-70.9)$ \\
Disease control rate $(\%, 95 \% \mathrm{CI})$ & $24(75.0,56.6-88.5)$ \\
\hline
\end{tabular}

CI confidence interval which exceeded the preestablished criterion and therefore met the anticipated RR. The disease control rate was 75.0\% (95\% CI: 56.6-88.5) with two patients achieving a CR (Table 2, Fig. 1). In the subset analysis by PS, for PS 2 (23 patients), the RR was $60.9 \%$ and DCR was $82.6 \%$. For PS 3-4 (10 patients), the RR was $30.0 \%$ and the DCR was $50.0 \%$.

The median PFS was 5.1 months (95\% CI: 3.55-6.67), the median OS was 10.0 months (95\% CI: 6.51-17.3) (Fig. 2), and the time to treatment failure was 4.3 months (95\% CI: 2.96-5.49). In the subset analysis by PS, for PS 2 , the median PFS and OS were 6.5 and 17.5 months, respectively. For PS 3-4, the median PFS and OS were 3.0 and 4.8 months, respectively. The PS improvement rate was $54.5 \%$ (95\% CI: 36.4-71.9, $P<0.001$ ), which is a good result (Fig. 3). A
Fig. 1 Waterfall plot of the best percentage change in target lesion size. $C R$ complete response, $N E$ non-evaluable, $P D$ progressive disease, $P R$ partial response, $S D$ stable disease

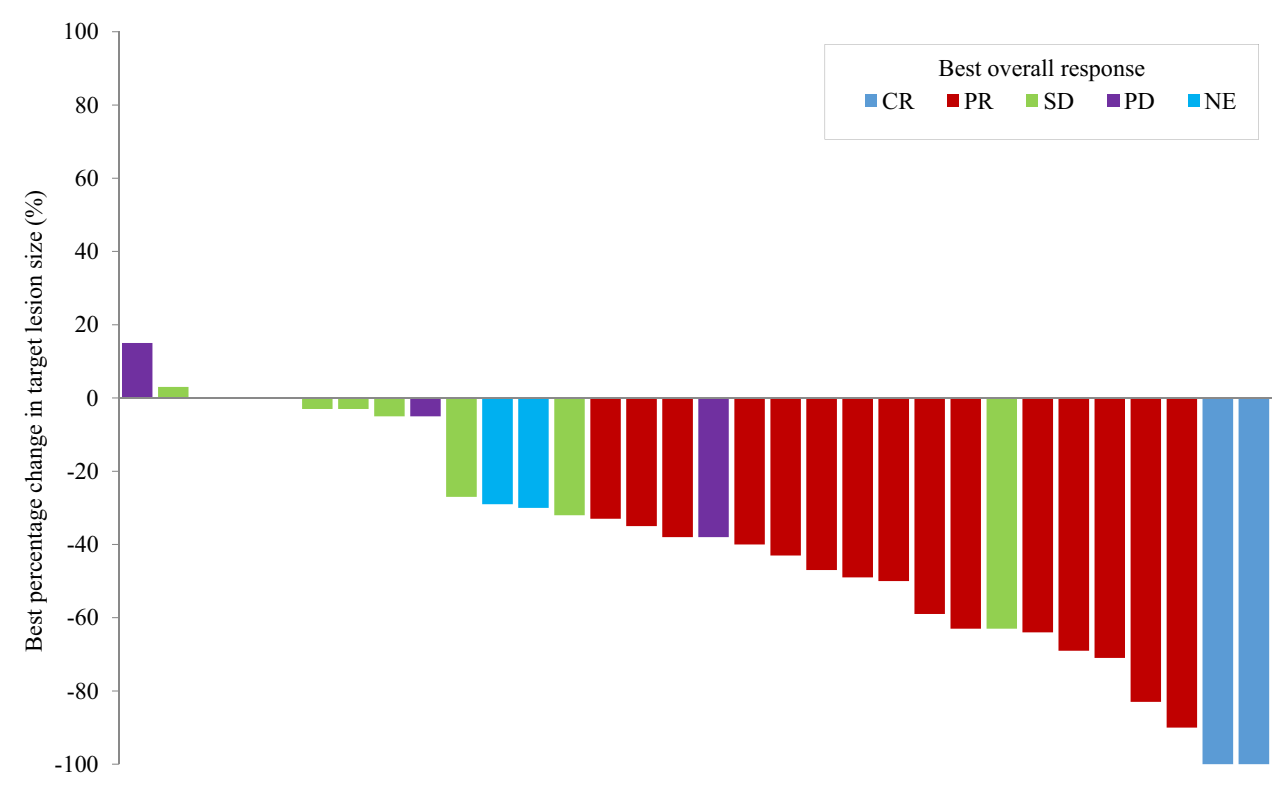

b

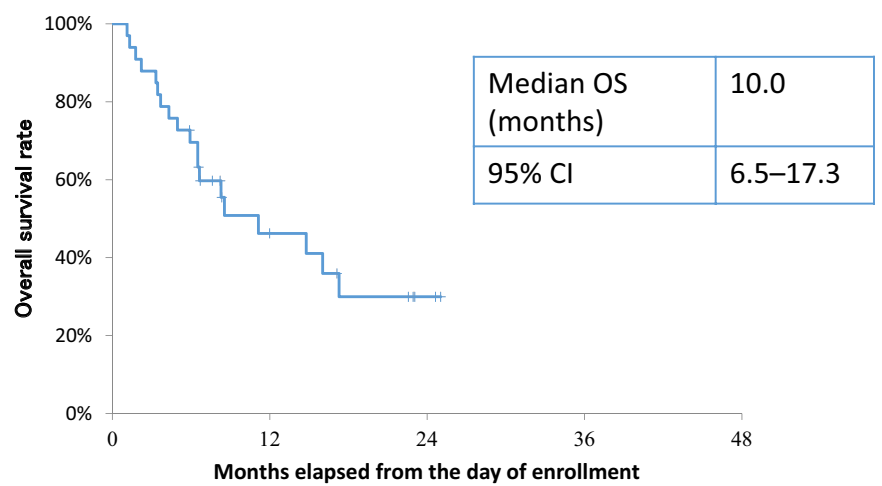

Fig. 2 Kaplan-Meier curves for progression-free survival (PFS) and overall survival (OS). a PFS, median observation period: 4.8 months, events occurred in $25 / 33$ patients. b OS, median observation period: 10.0 months, events occurred in $24 / 33$ patients. CI, confidence interval 


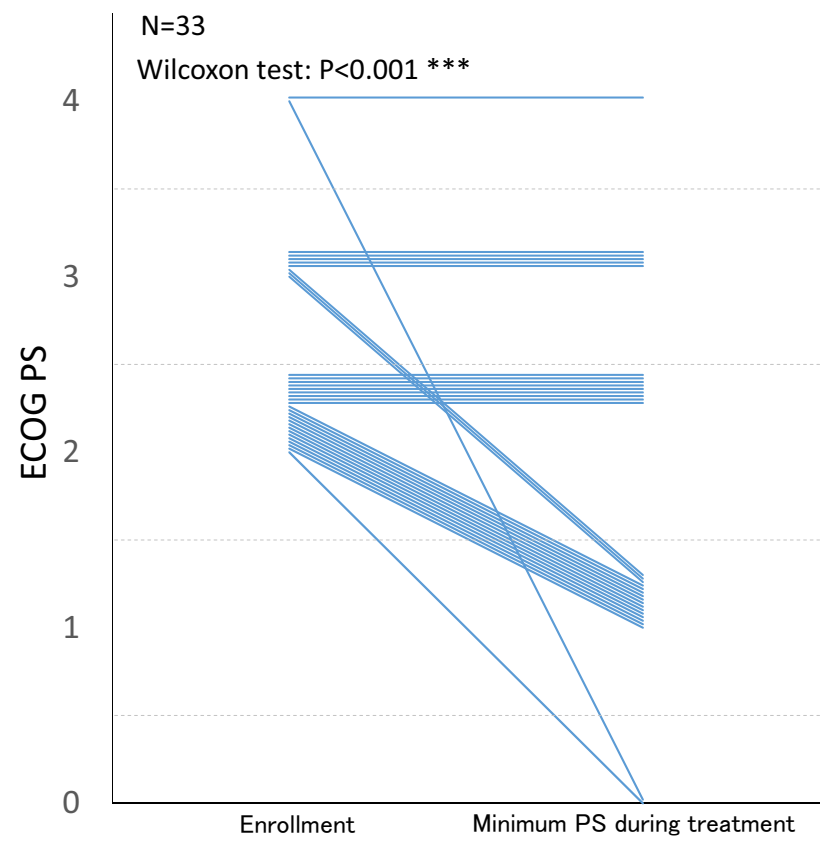

Fig. 3 Change in the performance status of each patient during treatment. Each line shows the change in performance status (PS) of a patient from baseline to their best status during the treatment (lowest observed PS from the day of the first dose to the day treatment was stopped). A clinically significant improvement was observed in 54.5\% (95\% CI 36.4-71.9) of patients. ECOG PS, Eastern Cooperative Oncology Group performance status

Table 3 Detection of EGFR mutations in plasma samples

\begin{tabular}{llll}
\hline & $\begin{array}{l}\text { Pre-treatment P0 } \\
(\%)\end{array}$ & $\begin{array}{l}\text { Under- } \\
\text { treatment } \\
\text { P1 }(\%)\end{array}$ & Post-PD P2 (\%) \\
\hline $\begin{array}{l}\text { Number of sam- } \\
\text { ples }\end{array}$ & 12 & 7 & 6 \\
$\begin{array}{c}\text { Activating muta- } \\
\text { tion }\end{array}$ & $11(91.7)$ & $3(42.9)$ & $4(66.7)$ \\
$\begin{array}{l}\text { Drug-resistant } \\
\text { mutation } \\
(\mathrm{T} 790 \mathrm{M})\end{array}$ & $12(100)$ & $2(28.6)$ & $3(50.0)$ \\
\hline
\end{tabular}

comparison of the smallest PS score during protocol treatment with the baseline PS score revealed no increase in PS in any patient.

\section{Plasma EGFR mutations}

The total number of collected plasma samples was 12 $(37.5 \%)$ at $\mathrm{P} 0,7(21.9 \%)$ at $\mathrm{P} 1$, and $6(18.8 \%)$ at $\mathrm{P} 2$ (Table 3). The frequencies of plasma-activating EGFR mutations and drug-resistant mutation (T790M) before the study treatment (P0) were $91.7 \%$ and $100 \%$, respectively. There was one case in which only T790M was detected without the activating EGFR mutations. Of the six cases positive for plasma-activating EGFR mutations at P0, three had no plasma mutations at P1 and three were still positive at P1 (Fig. 4). Among patients with detectable activating EGFR mutations at P0, the median PFS was longer for those in whom activating mutations were not detected at P1 than for those in whom the mutations were still detectable at P1 (15.0 vs. 4.3 months, respectively) (Fig. 5). Of the six cases positive for T790M mutation at P0, four had no T790M mutations at P1 and two were still positive at P1. The median PFS of T790M-negative cases at P1 was 11.2 months and that of the T790M-positive cases at P1 was 4.6 months (data not shown).

\section{Safety}

The most frequent adverse event of any grade was anemia $(81.8 \%)$, although anemia of grade 3 or greater severity occurred in just $6.1 \%$ of patients (Table 4 ). The most frequent adverse event of grade 3 or greater severity was lymphopenia at $12.1 \%$. ILD was reported in five cases $(15.2 \%)$, of which two were grade 3 or higher; one of these was a treatment-related death.

Dose reduction due to toxicity occurred in five patients (15.2\%). The reasons for this were increased aspartate transaminase and alanine transaminase levels, acne-like eruption, oral mucositis, purpura, urinary tract infection, and anorexia. Treatment was stopped in seven patients due to toxicity $(21.1 \%)$; the reasons were ILD, prolonged QT, corneal ulcer, inability to restart therapy after drug cessation, and discretion of the attending physician due to safety concerns.

\section{Discussion}

A few clinical studies have examined EGFR-TKI therapy in patients with EGFR-positive lung cancer and poor PS. Here, we confirmed that osimertinib is both efficacious and safe, which is beneficial for routine medical practice and therapeutic options for patients with T790M mutation-positive advanced NSCLC with EGFR-TKI resistance and poor PS, particularly for T790M-positive patients with no other treatment options.

This was a phase II investigator-initiated clinical trial conducted in patients who were T790M positive with a PS score of 2-4 and at least one failed EGFR-TKI treatment regimen. Although the median age of the participants was high at 72 years, and approximately $50 \%$ of the participants received at least their third-line therapy in this study, the RR of $53.1 \%$ was extremely good. The efficacy of osimertinib has previously been demonstrated in two clinical trials in patients with EGFR-positive lung cancer and good PS, where the 
Fig. 4 EGFR mutation status at different time points of osimertinib treatment. This figure depicts the percentage of each mutation at each time point of osimertinib treatment (P0, $\mathrm{P} 1$, and $\mathrm{P} 2$ ). The horizontal axis shows the number of patients. P0: plasma samples before the start of the study treatment, P1: plasma samples 8 weeks after the start of the study treatment, P2: plasma samples after disease progression

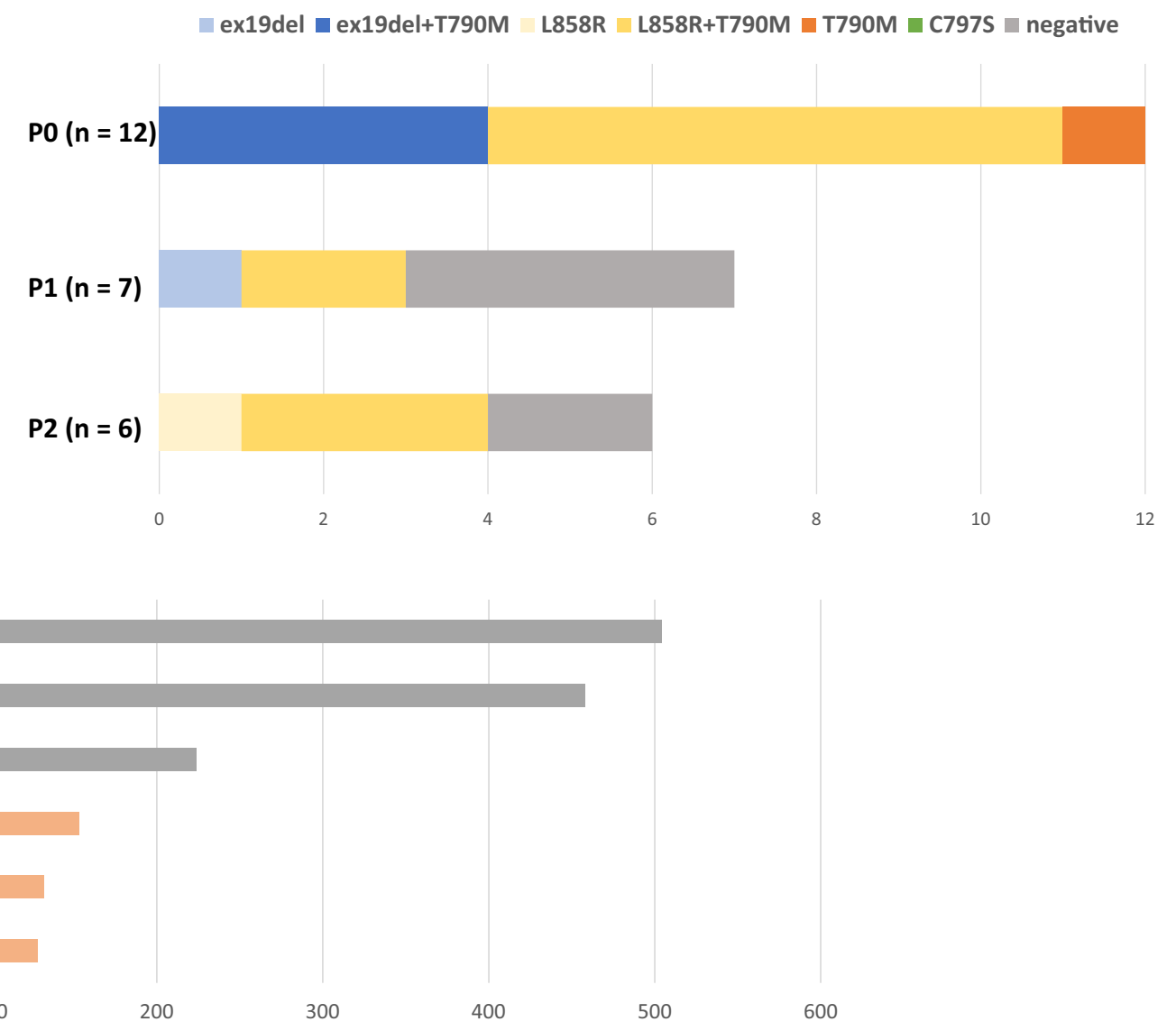

Fig. 5 Swimmer plot of progression-free survival (PFS) between patients with clearance and non-clearance of the activating EGFR mutations. Each histogram shows PFS. Gray histograms show the PFS of patients who experienced plasma clearance of activating mutations. Orange histograms show the PFS of patients with sus-

T790M variant was confirmed after treatment - the phase III AURA3 study that compared osimertinib with platinumdoublet chemotherapy (median age: 62 years) [10] and a pooled analysis of the phase II AURA and AURA2 studies (median age: 62 years) [14, 15]. In these clinical studies, the RR with osimertinib was $71 \%$ and $66 \%$, respectively. Meanwhile, Nakashima et al. reported a 53\% RR in prospective phase II study of poor PS [16]. The RR in our study not only met the study endpoint statistically but was also excellent considering that this study was conducted in older patients with poor PS. In the above two studies on good PS, the PFS with osimertinib was 10.1 and 9.9 months, respectively, which is substantially different from the 5.1 months observed in the present study. Among clinical studies that used the first-generation EGFR-TKI gefitinib, one study of first-line therapies in patients with EGFR-positive lung

\section{$\mathrm{PO}(+) \rightarrow \mathrm{P} 1(-) \mathrm{n}=3 \quad 15.0 \mathrm{M}$ $\mathrm{PO}(+) \rightarrow \mathrm{P} 1(+) \mathrm{n}=3 \quad 4.3 \mathrm{M}$}

tained plasma-activating mutations. P0: plasma samples before the start of the study treatment, P1: plasma samples 8 weeks after the start of the study treatment. (+): positive for plasma EGFR mutations, $(-)$ : negative for plasma EGFR mutations

cancer and good PS reported a PFS of 10.8 months [3], whereas another conducted in patients with poor PS reported a shorter PFS of 6.5 months [13]. Based on this, the different PS scores of patients enrolled in our study and other similar studies may be a major factor in their different clinical outcomes. We also suspect that clinical outcomes were affected by the differences in patient characteristics, such as older age, longer treatment history, and higher frequency of brain metastasis in our study.

In this study, we observed a PS improvement rate of $54.5 \%$ and PS scores that either remained the same or temporarily improved during treatment. We also observed a potential "Lazarus effect" in one patient whose PS score improved from 4 to 0 . Osimertinib is currently used as a first-line therapy for EGFR-positive cancer, because it is well indicated for cases that are EGFR-TKI-resistant and 
Table 4 Adverse events

\begin{tabular}{lll}
\hline Adverse event & All grades $(\%)$ & $\begin{array}{l}\text { Grade } 3 \text { or greater severity } \\
(\%)\end{array}$ \\
\hline Anemia & $27(81.8)$ & $2(6.1)$ \\
Hypoalbuminemia & $22(66.7)$ & $2(6.1)$ \\
Hyponatremia & $18(54.5)$ & $2(6.1)$ \\
Hypocalcemia & $15(45.5)$ & $1(3.0)$ \\
Increased ALP & $15(45.5)$ & $1(3.0)$ \\
Thrombopenia & $13(39.4)$ & 0 \\
Lymphopenia & $12(36.4)$ & $4(12.1)$ \\
Increased AST & $12(36.4)$ & $2(6.1)$ \\
Leukopenia & $11(33.3)$ & 0 \\
Increased ALT & $9(27.3)$ & $2(6.1)$ \\
Hyperkalemia & $9(27.3)$ & $1(3.0)$ \\
Increased creatinine & $9(27.3)$ & 0 \\
Proteinuria & $8(24.2)$ & 0 \\
Nail disorder & $7(21.2)$ & 0 \\
Hypertension & $6(18.2)$ & $1(3.0)$ \\
Rash & $5(15.2)$ & $1(3.0)$ \\
Acne-like eruption & $5(15.2)$ & $1(3.0)$ \\
Xerosis cutis & $5(15.2)$ & 0 \\
Anorexia & $5(15.2)$ & $3(9.1)$ \\
Interstitial lung disease & $5(15.2)$ & $2(6.1) ;$ G5 in 1 patient \\
Hypermagnesemia & $4(12.1)$ & $1(3.0)$ \\
Hypokalemia & $4(12.1)$ & 0 \\
Diarrhea & $4(12.1)$ & 0 \\
\hline
\end{tabular}

$A L P$ alkaline phosphatase, $A L T$ alanine transaminase, $A S T$ aspartate transaminase

T790M-positive, even when the patient has poor PS; however, rebiopsy must be seriously considered in patients whose first EGFR-TKI therapy is not osimertinib.

The safety evaluation in this study revealed anemia, lymphopenia, leukopenia, and other signs of myelosuppression, although all events were mild in severity and acceptable. Adverse events encountered with first- and second-generation EGFR-TKI therapies were eruption, diarrhea, and impaired liver function, but impaired liver function of grade 3 or greater severity only occurred in $6.1 \%$ of patients in this study. Nonetheless, one-fifth of patients in this study developed toxicity that required cessation of the treatment.

We also observed a high overall incidence of druginduced ILD at $15.2 \%$, with one case of death. Although the pooled analysis of the AURA and AURA2 studies showed a 3\% incidence of drug-induced ILD [15], an analysis of real-world data on osimertinib revealed that the incidence of drug-induced ILD was 6.8\% [17]. Given these findings, the relatively high incidence of drug-induced ILD observed in this study was likely due to the poor patients' PS, as well as ethnic differences, given that our study was conducted in Japanese patients. While there is no data on the efficacy and safety of osimertinib as the first-line therapy in patients with EGFR mutation-positive lung cancer and poor PS, based on the results of this study, caution should be paid to the possibility of drug-induced ILD when using osimertinib to treat patients with poor PS, even as a first-line therapy. Currently, osimertinib is used as a first-line therapy for EGFR-positive lung cancer.

Here, plasma-activating EGFR mutations and T790M mutation were detected at a high frequency using the PNALNA PCR clamp method before the study treatment. The high detection rate of EGFR mutations in this study might be related to the poor PS induced by high tumor burden in the patients. The plasma clearance of activating mutations during TKI treatment represents a potential predictive factor for response to TKI treatment [18-20].

Patients with EGFR-positive lung cancer are expected to respond to EGFR-TKI therapy, although almost all develop resistance 1-2 years after starting treatment. There are a variety of mechanisms of acquired resistance, with the T790M mutation reportedly accounting for $60 \%$ of patients who develop resistance [8]. Osimertinib can circumvent EGFR-TKI resistance, providing an excellent treatment option for patients in whom the T790M variant is confirmed after first- or second-generation EGFR-TKI therapy. This view is supported by the favorable response observed in this study, even among patients with poor PS. Our study was limited by the relatively small number of patients, and therefore, we could not conduct subgroup analysis. To enable the analysis of CNS reactions, we believe that it is necessary to focus on patients with CNS metastases in the future.

Osimertinib therapy exhibits acceptable efficacy in patients with T790M mutation-positive advanced NSCLC with EGFR-TKI resistance and poor PS; however, adverse events and ILD should be considered.

Acknowledgements We thank all our patients and their families, as well as all the site investigators. We thank Dr. Yasuo Saijo, Dr. Hiroyuki Shibata, and Dr Kenji Ohyama of the Safety Monitoring Committee, and Dr. Hirohisa Yoshizawa, Dr. Akinobu Yoshimura, and Dr. Yuichi Takiguchi of the Independent Efficacy Review Committee.

Funding This work was supported by AstraZeneca K.K. Only funding was provided, and AstraZeneca K.K. played no role in the clinical trial, from drafting and creating the protocol to managing study progress, collecting data, and performing statistical analyses.

Data availability The data obtained in this study were written in Japanese, and therefore, the raw data cannot be shared.

\section{Declarations}

Conflict of interest Y.T. received grants and personal fees from Daiichi Sankyo Co. Ltd. and AstraZeneca K.K. and personal fees from Chugai Pharmaceuticals Inc. outside the submitted work. A.N. received personal fees and research funding (institution) from AstraZeneca K.K., Chugai Pharmaceuticals Inc., MSD K.K., and Taiho Pharmaceuti- 
cal; personal fees from Nippon Boehringer Ingelheim Co. Ltd. and Novartis Pharma K.K.; and research funding (institution) from Ono Pharmaceutical, Bristol-Myers Squibb, Pfizer, Takeda Pharmaceutical, and Astellas Pharma Inc. outside the submitted work. H.Y. received personal fees from AstraZeneca K.K., Chugai Pharmaceuticals Inc., MSD K.K., Nippon Boehringer Ingelheim Co. Ltd., Taiho Pharmaceutical, Ono Pharmaceutical, Delta-Fly Pharma, Inc., Bristol-Myers Squibb Company, Novartis Pharma K.K., Kyowa Kirin Co., Ltd., Nippon Kayaku Co., Ltd., and Eli Lilly Japan K.K. outside the submitted work. S.O. received grants from Abbvie, Amgen, AstraZeneca, Bristol-Myers Squibb, Chugai Pharmaceutical, Kissei Pharmaceutical, Ono Pharmaceutical, Pfizer, Merck Biopharma, Sanofi, Taiho Pharmaceutical, and Takeda Pharmaceutical, and personal fees from AstraZeneca K.K. and Eli Lilly outside the submitted work. N.F. received personal fees from AstraZeneca K.K., Chugai Pharmaceutical, Nippon Boehringer Ingelheim Co. Ltd., Bristol-Myers Squibb Company, Eli Lilly Japan K.K., MSD K.K., Pfizer Japan Inc. Taiho Pharmaceutical, and Novartis Pharma K.K. outside the submitted work. E.I. received personal fees from AstraZeneca K.K. and Nippon Boehringer Ingelheim Co. Ltd. outside the submitted work. M.M. received personal fees from AstraZeneca K.K., Nippon Boehringer Ingelheim Co. Ltd., Chugai Pharmaceutical Co. Ltd., and Pfizer Japan Inc. outside the submitted work. S.M. received honoraria from AstraZeneca K.K., BristolMyers Squibb Company, Chugai Pharmaceutical Co. Ltd., Eli Lilly Japan K.K., MSD K.K., Nippon Boehringer Ingelheim Co. Ltd., Ono Pharmaceutical Co. Ltd., Pfizer Japan Inc., and Taiho Pharmaceutical Co. Ltd; advisory fees from Astellas Pharma Inc.; and research funding (institution) from Nippon Boehringer Ingelheim Co. Ltd. outside the submitted work. K.K. received personal fees from AstraZeneca K.K., Bristol-Myers Squibb, and Nippon Boehringer Ingelheim Co. Ltd. outside the submitted work. T.I. received grants and personal fees from Daiichi Sankyo Co. Ltd; personal fees from AstraZeneca K.K., Pfizer Japan Inc., and Nippon Boehringer Ingelheim Co., Ltd.; and grants from Pearl Therapeutics Inc. outside the submitted work.

Open Access This article is licensed under a Creative Commons Attribution 4.0 International License, which permits use, sharing, adaptation, distribution and reproduction in any medium or format, as long as you give appropriate credit to the original author(s) and the source, provide a link to the Creative Commons licence, and indicate if changes were made. The images or other third party material in this article are included in the article's Creative Commons licence, unless indicated otherwise in a credit line to the material. If material is not included in the article's Creative Commons licence and your intended use is not permitted by statutory regulation or exceeds the permitted use, you will need to obtain permission directly from the copyright holder. To view a copy of this licence, visit http://creativecommons.org/licenses/by/4.0/.

\section{References}

1. Kohno T, Nakaoku T, Tsuta K et al (2015) Beyond ALK-RET, ROS1 and other oncogene fusions in lung cancer. Transl Lung Cancer Res 4:156-164

2. Akamatsu H, Ninomiya K, Kenmotsu H et al (2019) The Japanese Lung Society guideline for non-small cell lung cancer, stage IV. Int J Clin Oncol 24:731-770

3. Maemondo M, Inoue A, Kobayashi K et al (2010) Gefitinib or chemotherapy for non-small cell lung cancer with mutated EGFR. N Engl J Med 362:2380-2388

4. Mitsudomi T, Morita S, Yatabe Y et al (2010) Gefitinib versus cisplatin plus docetaxel in patients with non-small-cell lung cancer harbouring mutations of the epidermal growth factor receptor (WJTOG3405): an open label, randomized phase 3 trial. Lancet Oncol 11:121-128
5. Yang JC-H, Wu Y-L, Schuler M et al (2015) Afatinib versus cisplatin-based chemotherapy for EGFR mutation-positive lung adenocarcinoma (LUX-Lung 3 and LUX-Lung 6): analysis of overall survival data from two randomised, phase 3 trials. Lancet Oncol 16:141-151

6. Hosomi Y, Morita S, Sugawara S et al (2020) Gefitinib alone versus gefitinib plus chemotherapy for non-small-cell lung cancer with mutated epidermal growth factor receptor: NEJ009 study. J Clin Oncol 38:115-123

7. Saito H, Fukuhara T, Furuya N et al (2019) Erlotinib plus bevacizumab versus erlotinib alone in patients with EGFR-positive advanced non-squamous non-small-cell lung cancer (NEJ026): interim analysis of an open-label, randomized, multicentre, phase 3 trial. Lancet Oncol 20:625-635

8. Yu HA, Arcila ME, Rekhtman N et al (2013) Analysis of tumor specimens at the time of acquired resistance to EGFR-TKI therapy in 155 patients with EGFR-mutant lung cancers. Clin Cancer Res 19:2240-2247

9. Kobayashi S, Boggon TJ, Dayaram T et al (2005) EGFR mutation and resistance of non-small-cell lung cancer to gefitinib. N Engl J Med 352:786-792

10. Mok TS, Wu Y-L, Ahn M-J et al (2017) Osimertinib or platinumpemetrexed in EGFR T790M-positive lung cancer. N Engl J Med 376:629-640

11. Soria J-C, Ohe Y, Vansteenkiste J et al (2018) Osimertinib in untreated EGFR-mutated advanced non-small-cell lung cancer. N Engl J Med 378:113-125

12. Goss G, Tsai C-M, Shepherd FA et al (2018) CNS response to osimertinib in patients with T790M-positive advanced NSCLC: pooled data from two phase II trials. Ann Oncol 29:687-693

13. Inoue A, Kobayashi K, Usui K et al (2009) First-line gefitinib for patients with advanced non-small-cell lung cancer harboring epidermal growth factor receptor mutations without indication for chemotherapy. J Clin Oncol 27:1394-1400

14. Hirashima T, Satouchi M, Hida T et al (2019) Osimertinib for Japanese patients with T790M-positive advanced non-small-cell lung cancer: a pooled subgroup analysis. Cancer Sci 110:2884-2893

15. Ahn MJ, Tsai CM, Shepherd FA et al (2019) Osimertinib in patients with T790M mutation-positive, advanced non-small cell lung cancer: long-term follow-up from a pooled analysis of 2 phase 2 studies. Cancer 125:892-901

16. Nakashima K, Ozawa Y, Daga H et al (2020) Osimertinib for patients with poor performance status and EGFR T790M mutation-positive advanced non-small cell lung cancer: a phase II clinical trial. Invest New Drugs 38:1854-1861

17. Ohe Y, Kato T, Sakai F et al (2020) Real-world use of osimertinib for epidermal growth factor receptor T790M-positive non-small cell lung cancer in Japan. Jpn J Clin Oncol 50:909-919

18. Fukuhara T, Saito H, Furuya N et al (2020) Evaluation of plasma EGFR mutation as an early predictor of response of erlotinib plus bevacizumab treatment in the NEJ026 study. EBioMedicine 57:102861

19. Iwama E, Sakai K, Hidaka N et al (2020) Longitudinal monitoring of somatic genetic alterations in circulating cell-free DNA during treatment with epidermal growth factor receptor-tyrosine kinase inhibitors. Cancer 126:219-227

20. Ebert EBF, McCulloch T, Hansen KH et al (2020) Clearing of circulating tumour DNA predicts clinical response to osimertinib in EGFR mutated lung cancer patients. Lung Cancer 143:67-72

Publisher's Note Springer Nature remains neutral with regard to jurisdictional claims in published maps and institutional affiliations. 


\section{Authors and Affiliations}

\section{Yukari Tsubata $^{1}$ (1) $\cdot$ Kana Watanabe $^{2} \cdot$ Ryota Saito $^{3} \cdot$ Atsushi Nakamura $^{4} \cdot$ Hiroshige Yoshioka $^{5} \cdot$ Mami Morita $^{2}$. Ryoichi Honda $^{6}$. Nobuhiro Kanaji ${ }^{7}$. Satoshi Ohizumi ${ }^{8}$. Daisuke Jingu ${ }^{9} \cdot$ Taku Nakagawa $^{10} \cdot$ Kensuke Nakazawa $^{11}$. Atsuto Mouri ${ }^{12}$. Susumu Takeuchi ${ }^{13} \cdot$ Naoki Furuya $^{14} \cdot$ Yuki Akazawa $^{15} \cdot$ Kiyotaka Miura $^{16} \cdot$ Eiki Ichihara $^{17}$. Makoto Maemondo ${ }^{18} \cdot$ Satoshi Morita ${ }^{19} \cdot$ Kunihiko Kobayashi $^{12} \cdot$ Takeshi Isobe $^{1}$}

1 Department of Internal Medicine, Division of Medical Oncology and Respiratory Medicine, Shimane University Faculty of Medicine, 89-1 Enya-cho, Izumo, Shimane 693-8501, Japan

2 Department of Respiratory Medicine, Miyagi Cancer Center, 47-1, Nodayama, Medeshimashiote, Natori, Miyagi 981-1293, Japan

3 Department of Respiratory Medicine, Tohoku University Hospital, 1-1, Seiryo-machi, Aoba-ku, Sendai, Miyagi 980-8574, Japan

4 Department of Pulmonary Medicine, Sendai Kousei Hospital, 4-15, Hirose-machi, Aoba-ku, Sendai, Miyagi 980-0873, Japan

5 Department of Thoracic Oncology, Kansai Medical University Hospital, 2-3-1, Shin-machi, Hirakata, Osaka 573-1191, Japan

6 Department of Respiratory Medicine, Asahi General Hospital, Asahi, Chiba 1326289-2511, Japan

7 Department of Internal Medicine, Division of Hematology, Rheumatology and Respiratory Medicine, Faculty of Medicine, Kagawa University, 1750-1, Ikenobe, Miki-cho, Kida-gun, Kagawa 761-0793, Japan

8 Department of Respiratory Medicine, National Hospital Organization Hokkaido Cancer Center, 2-3-54, 4-jyo, Kikusui, Shiraisi-ku, Sapporo, Hokkaido 003-0804, Japan

9 Department of Respiratory Medicine, Saka General Hospital, 16-5, Nishiki-cho, Shiogama, Miyagi 985-8506, Japan

10 Department of Thoracic Surgery, Omagari Kosei Medical Center, 8-65, Toori-machi, Omagari, Daisen, Akita 014-0027, Japan
11 Division of Clinical Medicine, Department of Pulmonary Medicine, Faculty of Medicine, University of Tsukuba, 2-1-1, Amakubo, Tsukuba, Ibaraki 305-8576, Japan

12 Department of Respiratory Medicine, Saitama Medical University International Medical Center, 1397-1, Yamane, Hidaka, Saitama 350-1298, Japan

13 Division of General Thoracic and Thyroid Surgery, Tokyo Medical University, 6-7-1, Nishi-shinjuku, Shinjuku, Tokyo 160-0023, Japan

14 Division of Respiratory Medicine, Department of Internal Medicine, St. Marianna University School of Medicine, 2-16-1, Sugao, Miyamae-ku, Kawasaki, Kanagawa 216-8511, Japan

15 Department of Thoracic Oncology, National Hospital Organization Osaka Toneyama Medical Center, 5-1-1, Toneyama, Toyonaka, Osaka 560-8552, Japan

16 Department of Respiratory Medicine, Shimane Prefectural Central Hospital, 4-1-1, Himehara, Izumo, Shimane 693-8555, Japan

17 Department of Allergy and Respiratory Medicine, Okayama University Hospital, 2-5-1, Shikata-cho, Kita-ku, Okayama 700-8558, Japan

18 Division of Pulmonary Medicine, Department of Internal Medicine, Iwate Medical University School of Medicine, 2-1-1, Idaidoori, Yahaba-cho, Shiwa-gun, Iwate 028-3695, Japan

19 Department of Biomedical Statistics and Bioinformatics, Kyoto University Graduate School of Medicine, 54, Shogoinkawahara-cho, Sakyo-ku, Kyoto 606-8507, Japan 